\title{
Obtaining triplet from quaternions
}

\author{
Ali Atasoy ${ }^{a *}$, Yusuf Yayli ${ }^{b}$ \\ ${ }^{a}$ Keskin Vocational School, Kırıkkale University, Turkey \\ ${ }^{b}$ Department of Mathematics, Ankara University, Turkey \\ aliatasoy@kku.edu.tr,yayli@science.ankara.edu.tr
}

\section{ARTICLE INFO}

Article history:

Received: 24 August 2019

Accepted: 10 May 2020

Available Online: 28 January 2021

\section{Keywords:}

Dual quaternion

Real quaternion

Triplet

Rotation

Screw operator

AMS Classification 2010

11R52, 51M99

\begin{abstract}
In this study, we obtain triplets from quaternions. First, we obtain triplets from real quaternions. Then, as an application of this, we obtain dual triplets from the dual quaternions. Quaternions, in many areas, it allows ease in calculations and geometric representation. Quaternions are four dimensions. The triplets are in three dimensions. When we express quaternions with triplets, our study is conducted even easier. Quaternions are very important in the display of rotational movements. Dual quaternions are important in the expression of screw movements. Reducing movements from four dimensions to three dimensions makes our study easier. This simplicity is achieved by obtaining triplets from quaternions.
\end{abstract}

\section{Introduction}

A real quaternion is defined that

$$
Q=w+x i+y j+z k
$$

where $w, x, y, z$ are real numbers and

$$
\begin{gathered}
i^{2}=j^{2}=k^{2}=i j k=-1 \\
i j=k, j k=i, k i=j \\
j i=-k, k j=-i, i k=-j .
\end{gathered}
$$

The norm of a real quaternion $Q$ is

$$
|Q|^{2}=Q \bar{Q}=w^{2}+x^{2}+y^{2}+z^{2} .
$$

The set of quaternions is indicated by $H$.

A dual quaternion $\hat{Q}$ is defined by

$$
\widehat{Q}=\widehat{w}+\hat{x} i+\hat{y} j+\hat{z} k
$$

where $\widehat{w}, \hat{x}, \hat{y}, \hat{z} \in \widehat{D}(\widehat{D}$ is dual number set). If $\hat{x} \in \widehat{D}$ then

$$
\hat{x}=x+\varepsilon x^{*}
$$

where $x, x^{*} \in R$ ( $R$ is real number set) and $\varepsilon^{2}=0$. Thus, we can write

$$
\hat{Q}=Q+\varepsilon Q^{*},
$$

where $Q$ is real quaternion component and $Q^{*}$ is pure dual quaternion component. The norm of a dual quaternion $\hat{Q}$ is

$$
\begin{aligned}
& |\hat{Q}|^{2}=\hat{Q} \overline{\hat{Q}} \\
& =\left(Q+\varepsilon Q^{*}\right)\left(\bar{Q}+\varepsilon \bar{Q}^{*}\right) \\
& =Q \bar{Q}+\varepsilon\left(Q \bar{Q}^{*}+Q^{*} \bar{Q}\right) \\
& \quad=\widehat{w}^{2}+\hat{x}^{2}+\hat{y}^{2}+\hat{z}^{2} .
\end{aligned}
$$

The dual quaternion set is indicated by $\widehat{H}[3]$.

The triplets is in a three-dimensional space. They can be obtained from arbitrary quaternions in fourdimensional space. So, we can make our study easier.

\section{Triplets and real quaternions}

The polar form of a quaternion containing complex module and complex argument was expressed by Sangwine and Bihan (see [1] for detailed information) Let's take

$$
Q=w+x i+y j+z k
$$

real quaternion. Let

$$
A=|Q| \frac{\zeta}{|\zeta|}
$$

where $\zeta=w+x i . Q$ can be expressed as

$$
Q=A e^{B j}
$$


where $B=c+d i$ is a complex number. Simply,

$$
e^{B j}=\alpha+\beta j+\gamma k
$$

triplet is obtain by multiplying on the left by $\hat{A}^{-1}$ of $\hat{Q}$.

It is known that

$$
B j=(c+d i) j=c j+d k
$$

and

$$
|B j|=|B| .
$$

Then it can be written that

$$
B j=|B j| \frac{B j}{|B j|}=|B| \frac{B j}{|B|} .
$$

Accordingly,

$$
\begin{aligned}
& e^{B j}=e^{|B| \frac{B j}{|B|}} \\
& =\cos |B|+\frac{B j}{|B|} \sin |B| \\
& =\cos |B|+\frac{c}{|B|} \sin |B| j+\frac{d}{|B|} \sin |B| k \\
& =\alpha+\beta j+\gamma k
\end{aligned}
$$

where $\alpha=\cos |B|, \beta=\frac{c}{|B|} \sin |B|, \gamma=\frac{d}{|B|} \sin |B|$ (for detailed information see [1, 3, 4]). We can write

$$
q=e^{B j}=e^{|B| \frac{B j}{|B|}}=e^{\xi j_{\varphi}}
$$

where $|B|=\xi, \frac{B j}{|B|}=j_{\varphi}$. Accordingly, we can express $Q$ quaternion with $Q=A q$ where $A$ is a complex number and $q$ is a triplet. Reverse the process, let's take $\alpha+\beta j+\gamma k$ unit quaternion. Since $\cos |B|=\alpha$ equality, $|B|=\arccos \alpha$ and $\sin |B|=\sqrt{1-\alpha^{2}}$. Then $B=c+d i$ can be writen where $c=\frac{|B| \beta}{\sin |B|}, d=\frac{|B| \gamma}{\sin |B|}$.

A leaf is determined with a unit vector $j_{\varphi}$. This vector makes a angle $\varphi$ with the positive $j$ direction in the $(j, k)$-plane. This unit vector can be expressed as

$$
j_{\varphi}=(\cos \varphi) j+(\sin \varphi) k
$$

and $j_{\varphi}^{2}=-1$. This is a pure unit quaternion. In this leaf, let triplet $q$ be a non-zero. This leaf is spanned by 1 and $j_{\varphi}$. We can write $q$ in terms of its components along these vectors. Namely, we can write that

$$
e^{B j}=q=(\cos \xi) 1+(\sin \xi) j_{\varphi}=e^{\xi j_{\varphi}}
$$

where $\xi$ be the angle that $q$ makes with the positive vector 1 direction in the leaf.

Thus, with the help of equation $B j=\xi j_{\varphi}, B$ can be calculated $[2,5]$. According to Figure $1, q \in S p\left\{1, j_{\varphi}\right\}$.

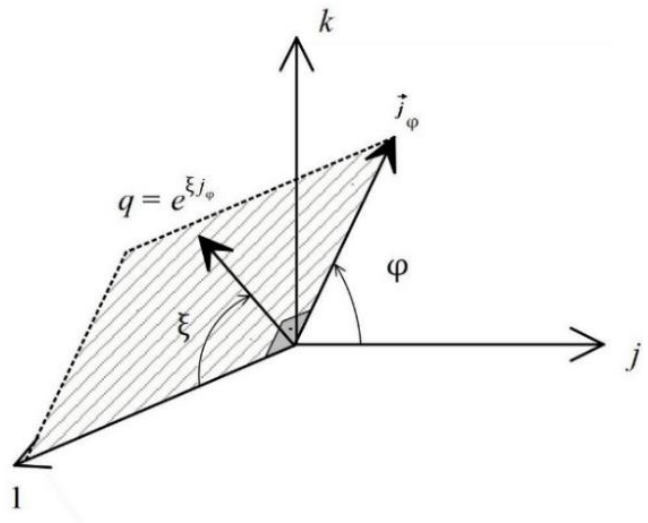

Figure 1. Triplet $q$.

Example 1. Consider $X=\frac{1}{\sqrt{3}}+\frac{1}{\sqrt{3}} j+\frac{1}{\sqrt{3}} k$. Then

$$
\begin{array}{r}
X=\frac{1}{\sqrt{3}}+\frac{\sqrt{2}}{\sqrt{3}}\left(\frac{1}{\sqrt{2}} j+\frac{1}{\sqrt{2}} k\right) \\
=\cos \xi+(\sin \xi) j_{\varphi} \\
=e^{\xi j_{\varphi}}
\end{array}
$$

where $j_{\varphi}=\frac{1}{\sqrt{2}} j+\frac{1}{\sqrt{2}} k, \cos \xi=\frac{1}{\sqrt{3}}$ and $\sin \xi=\frac{\sqrt{2}}{\sqrt{3}}$. Then $|B|=\xi=\arccos \left(\frac{1}{\sqrt{3}}\right)$. So,

$$
\xi j_{\varphi}=B j
$$

$$
\begin{aligned}
=\arccos \left(\frac{1}{\sqrt{3}}\right)\left(\frac{1}{\sqrt{2}} j+\frac{1}{\sqrt{2}} k\right) \\
=\left(\frac{1}{\sqrt{2}} \arccos \left(\frac{1}{\sqrt{3}}\right)+\frac{1}{\sqrt{2}} \arccos \left(\frac{1}{\sqrt{3}}\right) i\right) j
\end{aligned}
$$

and

$$
B=\frac{1}{\sqrt{2}} \arccos \left(\frac{1}{\sqrt{3}}\right)+\frac{1}{\sqrt{2}} \arccos \left(\frac{1}{\sqrt{3}}\right) i .
$$

\section{Dual triplets and dual quaternions}

Similar to the polar form of real quaternions, we can also express the polar form of dual quaternions.

Theorem 1. Let's take

$$
\hat{Q}=\widehat{w}+\hat{x} i+\hat{y} j+\hat{z} k
$$

dual quaternion. Let

$$
\hat{A}=|\hat{Q}| \frac{\hat{\zeta}}{|\hat{\zeta}|}
$$

where $\hat{\zeta}=\widehat{w}+\hat{x} i . \hat{Q}$ can be written in the form

$$
\hat{Q}=\hat{A} e^{\hat{B} j}
$$

where $\hat{B}=\hat{c}+\hat{d} i$ is a dual complex number.

Proof. Simply, 


$$
e^{\hat{B} j}=\hat{\alpha}+\hat{\beta} j+\hat{\gamma} k
$$

triplet is obtain by multiplying on the left by $\hat{A}^{-1}$ of $\hat{Q}$. It is known that

$$
\hat{B} j=(\hat{c}+\hat{d} i) j=\hat{c} j+\hat{d} k
$$

and $|\hat{B} j|=|\hat{B}|$. Then it can be written that

$$
\hat{B} j=|\hat{B} j| \frac{\hat{B} j}{|\hat{B} j|}=|\hat{B}| \frac{\hat{B} j}{|\hat{B}|} .
$$

Accordingly,

$$
\begin{aligned}
& e^{\hat{B} j}=e^{|\hat{B}| \frac{\hat{B} j}{|\hat{B}|}} \\
& =\cos |\hat{B}|+\frac{\widehat{B} j}{|\hat{B}|} \sin |\hat{B}| \\
& =\cos |\hat{B}|+\frac{\hat{c}}{|\hat{B}|} \sin |\hat{B}| j+\frac{\hat{d}}{|\hat{B}|} \sin |\hat{B}| k \\
& =\hat{\alpha}+\hat{\beta} j+\hat{\gamma} k
\end{aligned}
$$

where $\hat{\alpha}=\cos |\hat{B}|, \hat{\beta}=\frac{\hat{c}}{|\hat{B}|} \sin |\hat{B}|, \gamma=\frac{\hat{d}}{|\hat{B}|} \sin |\hat{B}|$ (see [1], [3] and [4]). We can write

$$
\hat{q}=e^{\hat{B} j}=e^{|\hat{B}| \frac{\hat{B} j}{|\widehat{B}|}}=e^{\hat{\xi} j_{\widehat{\varphi}}}
$$

where $|\hat{B}|=\hat{\xi}, \frac{\hat{B} j}{|\hat{B}|}=j_{\widehat{\varphi}}$.

Accordingly, we can express $\hat{Q}$ dual quaternion with

$$
\hat{Q}=\hat{A} \hat{q}
$$

where $\hat{A}$ and $\hat{q}$ are dual complex number and dual triplet respectively.

Reverse the process, let's take $\hat{\alpha}+\hat{\beta} j+\hat{\gamma} k$ unit quaternion. Since $\cos |\hat{B}|=\hat{\alpha}$ equality, $|\hat{B}|=\arccos \hat{\alpha}$ and $\sin |\hat{B}|=\sqrt{1-\hat{\alpha}^{2}}$. Then $\hat{B}=\hat{c}+\hat{d} i$ can be writen where

$$
\hat{c}=\frac{|\hat{B}| \hat{\beta}}{\sin |\hat{B}|}=\frac{(\arccos \hat{\alpha}) \hat{\beta}}{\sin (\arccos \hat{\alpha})}
$$

and

$$
\hat{d}=\frac{|\hat{B}| \hat{\gamma}}{\sin |\hat{B}|}=\frac{(\arccos \hat{\alpha}) \hat{\gamma}}{\sin (\arccos \hat{\alpha})} .
$$

A leaf is determined by a unit dual vector $j_{\widehat{\varphi}}$. This dual vector make a dual angle $\hat{\varphi}$ with the positive $j$ direction in the $(j, k)$-plane making. This unit vector can be expressed as

$$
j_{\widehat{\varphi}}=(\cos \hat{\varphi}) j+(\sin \hat{\varphi}) k
$$

and $j_{\widehat{\varphi}}^{2}=-1$. This is a pure unit dual quaternion. In this leaf, let triplet $\hat{q}$ be a non-zero. This leaf is spanned by 1 and $j_{\hat{\varphi}}$. We can write $\hat{q}$ in terms of its components along these vectors. Namely, we can write that

$$
e^{\hat{B} j}=\hat{q}=(\cos \widehat{\xi}) 1+(\sin \widehat{\xi}) j_{\widehat{\varphi}}=e^{\widehat{\xi} j_{\widehat{\varphi}}} .
$$

where $\hat{\xi}$ be the dual angle that $\hat{q}$ makes with the positive vector 1 direction in the leaf.

Thus, with the help of equation $\hat{B} j=\hat{\xi} j_{\hat{\varphi}}, \hat{B}$ can be calculated [2, 5]. According to the Figure 2, $\hat{q} \in$ $S p\left\{1, j_{\hat{\varphi}}\right\}$ and $\hat{A} \in S p\{1, i\}$.

For every unit dual quaternion, we can write that

$\hat{Q}=\cos \hat{\theta}+\hat{\mu} \sin \hat{\theta}$

$=\cos \left(\theta+\varepsilon \theta^{*}\right)+\hat{\mu} \sin \left(\theta+\varepsilon \theta^{*}\right)$

where $\theta$ is rotation angle and $\theta^{*}$ is translation component about the dual axis $\hat{\mu}$. Accordingly, $\hat{Q}$ is a screw operator. Because $\hat{Q}$ makes dual angular displacement about dual vector axis [3].

From [6], the dual angle $\hat{\theta}=\theta+\varepsilon \theta^{*}$ makes rotation as $\theta$ and translation as $\theta^{*}$ about the dual axis $\hat{\mu}$ where

$$
\cos \hat{\theta}=\cos \theta-\varepsilon \theta^{*} \sin \theta
$$

and

$$
\sin \hat{\theta}=\sin \theta+\varepsilon \theta^{*} \cos \theta .
$$

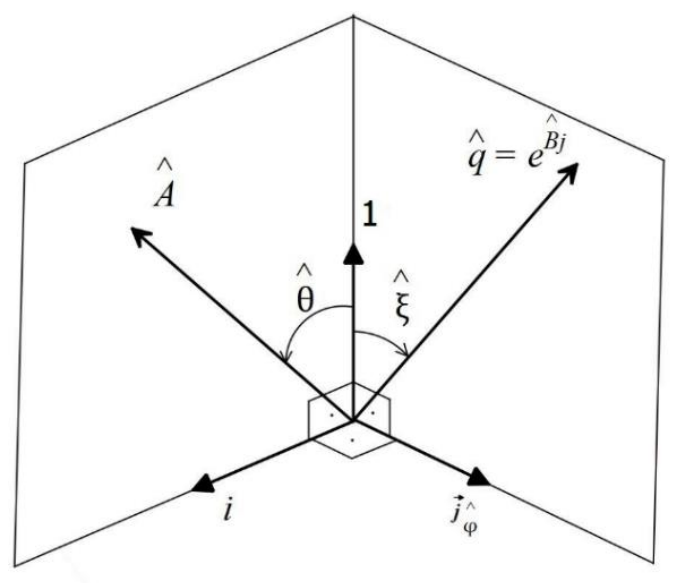

Figure 2. Dual triplet $\hat{q}$ and dual complex number $\hat{A}$.

Example 2. Let $\hat{Q}=(1+\varepsilon) i+(1-\varepsilon) j+k$ then $|\hat{Q}|=\sqrt{3}=|\hat{A}|$ where

$$
\hat{A}=\sqrt{3} \frac{(1+\varepsilon) i}{1+\varepsilon}=\sqrt{3} i
$$

and

$$
\hat{A}^{-1}=\frac{-\sqrt{3} i}{3}=\frac{-i}{\sqrt{3}} .
$$

So,

$$
\begin{aligned}
& \hat{Q}=\widehat{A} e^{\hat{\xi} j_{\widehat{\varphi}}} \\
& (1+\varepsilon) i+(1-\varepsilon) j+k=\sqrt{3} i e^{\hat{\xi} j_{\widehat{\varphi}}} .
\end{aligned}
$$


Let multiply $\hat{Q}$ by the inverse of $\hat{A}$ on the left. Then

$\hat{q}=e^{\hat{\xi}} j_{\hat{\varphi}}$

$=\frac{-i}{\sqrt{3}}[(1+\varepsilon) i+(1-\varepsilon) j+k]$

$=\frac{1+\varepsilon}{\sqrt{3}}+j_{\widehat{\varphi}} \frac{\sqrt{2-2 \varepsilon}}{\sqrt{3}}$

and

$\cos \hat{\xi}=\frac{1+\varepsilon}{\sqrt{3}}$

$\sin \hat{\xi}=\frac{\sqrt{2-2 \varepsilon}}{\sqrt{3}}$

where $\hat{\xi}$ is dual angle. So,

$$
\begin{gathered}
\cos \hat{\xi}=\cos \left(\xi+\varepsilon \xi^{*}\right)=\cos \xi-\varepsilon \xi^{*} \sin \xi \\
=\frac{1}{\sqrt{3}}+\varepsilon \frac{1}{\sqrt{3}}
\end{gathered}
$$

where $\cos \xi=\frac{1}{\sqrt{3}}, \sin \xi=\frac{-\sqrt{2}}{\sqrt{3}}, \xi^{*}=\frac{1}{\sqrt{2}}$ and

$$
\hat{\xi}=\arccos \left(\frac{1}{\sqrt{3}}+\varepsilon \frac{1}{\sqrt{3}}\right) \text {. }
$$

Furthermore, we can write that

$$
\begin{aligned}
& j_{\widehat{\varphi}}=\frac{\frac{1}{\sqrt{3}} j-\frac{1-\varepsilon}{\sqrt{3}} k}{\frac{\sqrt{2-2 \varepsilon}}{\sqrt{3}}} \\
& =\frac{j-(1-\varepsilon) k}{\sqrt{2-2 \varepsilon}}
\end{aligned}
$$

and

$$
\begin{aligned}
& \hat{B} j=\hat{\xi} j_{\widehat{\varphi}} \\
& =\arccos \left(\frac{1}{\sqrt{3}}+\varepsilon \frac{1}{\sqrt{3}}\right)\left(\frac{j-(1-\varepsilon) k}{\sqrt{2-2 \varepsilon}}\right) \\
& =\left[\arccos \left(\frac{1}{\sqrt{3}}+\varepsilon \frac{1}{\sqrt{3}}\right)\left(\frac{1-(1-\varepsilon) i}{\sqrt{2-2 \varepsilon}}\right)\right] j .
\end{aligned}
$$

Thus, $\hat{B}=\arccos \left(\frac{1}{\sqrt{3}}+\varepsilon \frac{1}{\sqrt{3}}\right)\left(\frac{1-(1-\varepsilon) i}{\sqrt{2-2 \varepsilon}}\right)$. Checking the result:

$$
\begin{aligned}
& \hat{A} \hat{q}=\hat{A} e^{\hat{\xi} j_{\hat{\varphi}}} \\
& \left.=\sqrt{3} i \exp \left[\arccos \left(\frac{1}{\sqrt{3}}+\varepsilon \frac{1}{\sqrt{3}}\right) j \frac{j-(1-\varepsilon) k}{\sqrt{2-2 \varepsilon}}\right)\right] \\
& =\sqrt{3} i\left(\frac{1+\varepsilon}{\sqrt{3}}+\frac{1}{\sqrt{3}} j+\frac{\varepsilon-1}{\sqrt{3}} k\right) \\
& =(1+\varepsilon) i+(1-\varepsilon) j+k \\
& =\hat{Q} .
\end{aligned}
$$

\section{Conclusion}

Here, triplets are obtained from the real quaternions. Then, dual triplets are obtained from the dual quaternions. Thus, it is believed that the quaternions which have an important place in motion geometry in general are made more useful. This will contribute to the understanding of some concepts such as rotation, translation, displacement and screw movement.

We know that a $Q$ unit real quaternion is rotation operator. Thus, a unit quaternion can be expressed by two rotation operators.

The displacement of a rigid body is screw displacement. This displacement can be made with screw operator. Every $\hat{Q}$ unit dual quaternion is screw operator. A unit dual quaternion $\hat{Q}$ can be expressed as $\hat{Q}=\hat{A} \hat{q}$, where $\hat{A}$ is a unit dual complex number and $\hat{q}$ is a unit dual triplet. Thus, two screw operators can be expressed with a unit dual quaternion.

\section{References}

[1] Sangwine, S.J., \& Bihan, N.L. (2010). Quaternion polar representation with a complex modulus and complex argument inspired by the CayleyDickson form. Advanced Applied Clifford Algebra, (20), 111-120.

[2] Pfaff, F.R. (2000). A commutative multiplication of number triplets. The American Mathematical Monthly 107(2), 156-162.

[3] Akyar, B. (2008). Dual quaternions in spatial kinematics in an algebraic sense. Turkish Journal of Mathematics (32), 373-391.

[4] Dimentberg, F.M. (1965). The screw calculus and its applications in mechanics; Foreign Division Translation FTD-HT-23-1632-67.

[5] Kula, L., \& Yaylı, Y. (2006). A commutative multiplication of dual number triplets. Journal of Science of Dumlupinar University (10), 53-60.

[6] Hanson, A.J. (2005). Visualizing quaternion, Morgan-Kaufmann, Elsevier.

Ali Atasoy received his undergraduate degree in mathematics education from the Ankara University in 1995. He has completed his M.Sc. at Ankara University and Ph.D. degree at Dumlupinar University in geometry. He has been working as a faculty member at Keskin Vocational School, Kirlkkale University. His research areas are quaternions and their applications.

https://orcid.org/0000-0002-1894-7695

Yusuf Yayl received his undergraduate degree in 1983 from Inönü University in mathematics. He has completed his M.Sc. and Ph.D. degrees at Gazi University. He has been working as Professor at the Department of Mathematics, Ankara University. His research areas are quaternions and differential geometry.

https://orcid.org/0000-0003-4398-3855 
An International Journal of Optimization and Control: Theories \& Applications (http://ijocta.balikesir.edu.tr)

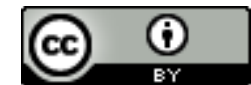

This work is licensed under a Creative Commons Attribution 4.0 International License. The authors retain ownership of the copyright for their article, but they allow anyone to download, reuse, reprint, modify, distribute, and/or copy articles in IJOCTA, so long as the original authors and source are credited. To see the complete license contents, please visit http://creativecommons.org/licenses/by/4.0/. 